\title{
ULTRA-LOW ENERGY STORAGE RING AT FLAIR
}

\author{
O. Gorda ${ }^{1}$, J. Harasimowicz ${ }^{3}$, O. Karamyshev ${ }^{1,2}$, G. Karamysheva ${ }^{1,2}$, D. Newton ${ }^{3}$, A. Papash ${ }^{1,2}$, \\ M. Panniello ${ }^{1}$, M. Putignano ${ }^{3}$, M. Siggel-King ${ }^{3}$, A. Smirnov ${ }^{1,2}$ and C.P. Welsch ${ }^{3, \#}$ \\ ${ }^{1}$ Max Planck Institute for Nuclear Physics, Heidelberg, Germany \\ ${ }^{2}$ JINR, Dubna, Russia (on leave) \\ ${ }^{3}$ Cockcroft Institute and the University of Liverpool, UK
}

\section{Abstract}

The Ultra-low energy electrostatic Storage Ring (USR) at the future Facility for Low-energy Antiproton and Ion Research (FLAIR) will provide cooled beams of antiprotons in the energy range between $300 \mathrm{keV}$ down to $20 \mathrm{keV}$. Based on the original design concept developed in 2005, the USR has been completely redesigned over the past few years. The ring structure is now based on a 'split achromat' lattice. This ensures compact ring dimensions of $10 \times 10 \mathrm{~m}^{2}$, whilst allowing both, in-ring experiments with gas jet targets and studies with extracted beams.

In the USR, a wide range of beam configurations will be provided, ranging from very short pulses in the nanosecond regime to a coasting beam. The ring also features a combined fast and slow extraction scheme that can provide external experiments with cooled beams of different time structure. Furthermore, studies into beam diagnostics methods for monitoring ultra-low energy ions at beam intensities of less than $10^{6}$ were carried out. Here, we present the final USR design with an emphasis on the expected beam parameters available to the experiments at FLAIR.

\section{INTRODUCTION}

Antiprotons stored and cooled at low energies in a storage ring or at rest in traps are highly desirable for the investigation of a large number of basic questions on fundamental interactions, on the static structure of exotic antiprotonic atomic systems and (radioactive) nuclei and on the time-dependent quantum dynamics of correlated systems $[1,2]$.

Studies of fundamental interactions include for example CPT measurement of the $1 \mathrm{~s}-2 \mathrm{~s}$ transition or of the ground-state hyperfine structure of antihydrogen, by highresolution spectroscopy, with accuracies similar to the ones achieved for hydrogen $\left(10^{-14}[3]\right.$ and $10^{-12}[4,5]$, resp.) as well as gravity experiments with antimatter [6]. Structure measurements extend from few-particle QED, correlation, and relativistic effects in antiprotonic atoms, to the investigation of the low-energy limit, i.e. of nonperturbative QCD and to nuclear skins exploration.

In addition, low-energy antiprotons might be the ideal and perhaps the only tool to study in detail correlated quantum dynamics of few-electron systems in the femtoand sub-femtosecond time regime [7]. Advanced storage

\footnotetext{
\#c.p.welsch@liverpool.ac.uk
}

ring and detection technologies in combination will enable, for the first time, access to kinematically complete antiproton-induced rearrangement and fragmentation measurements. These benchmark measurements will ultimately lead to a resolution of contradicting predictions by existing theories and explicitly address the few-body Coulomb problem - one of the most fundamental, yet unsolved problems in physics.

To enable the efficient investigation of essentially all of these important questions in detail, a novel electrostatic cooler synchrotron has been developed by the QUASAR group. The aim will be to slow down antiprotons to very low energies between 20 and $300 \mathrm{keV}$. This will provide a world-wide unique facility for both in-ring studies, with intensities of up to $10^{12}$ cooled and stored antiprotons per second, as well as for experiments requiring extracted slow beams.

In order to match the requirements from the envisaged experiments, different modes of operation had to be included in the USR design: deceleration and electron cooling, fast and slow extraction, ultra-short bunches for in-ring experiments, and optimization of the beam shape, size, and dispersion for the respective internal or external experiment.

\section{RING LAYOUT}

The final layout of the USR Lattice is based on a splitachromat geometry, which gives the necessary flexibility to satisfy the before-mentioned boundary conditions and is illustrated in Fig.1. The USR has a circumference of $42.6 \mathrm{~m}$, and thus almost unaltered geometrical dimensions as compared to the initial design [8]. Four $4 \mathrm{~m}$ long straight sections are used to accommodate a Reaction Microscope (ReMi), the different rf systems for the short bunch operation mode [9], the electron cooler, the decelerating drift tube, and the elements for fast/slow extraction [10]. One of the straight sections is kept free for the possible inclusion of a merged positron ring. Five electrostatic quadrupoles, two $8^{\circ}$ and two $37^{\circ}$ electrostatic deflectors form an achromatic $90^{\circ}$ bend. A drift space of 1 $\mathrm{m}$ between the deflectors is left to allow for the detection of neutral particles leaving the ring with an opening angle of up to $\Omega= \pm 0.7^{\circ}$ after the ReMi.

The linear machine lattice was developed with the computer codes MAD-X and Trace3D and details can be found in [11]. The quadrupole in the middle of each bending section is used to modify the beam characteristics to either provide a round beam mode or to achieve 
achromatic conditions, where both the dispersion function and its first derivative are zero.

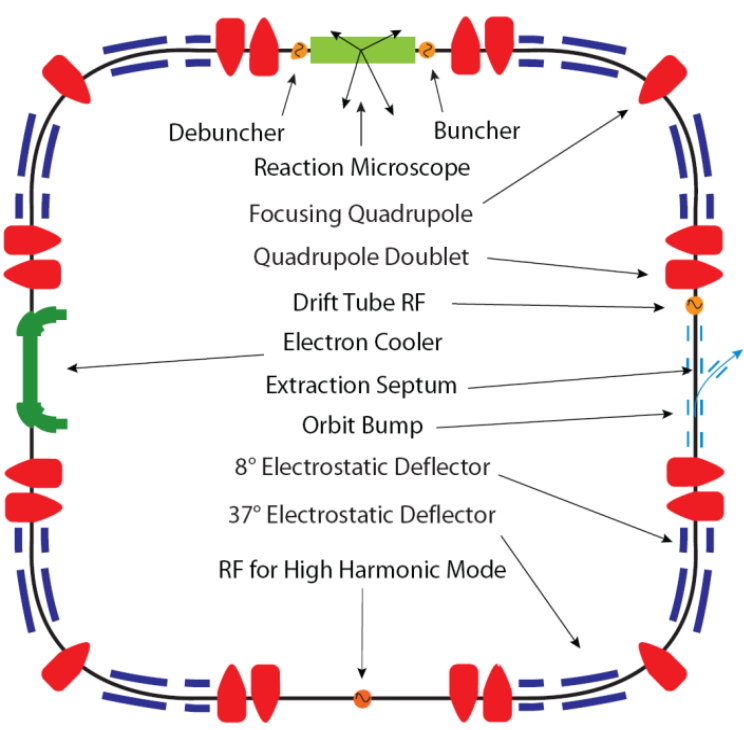

Figure 1: Layout of the ultra-low energy storage ring.

\section{Beam Dynamics}

As essentially all accelerator design codes do not foresee the use of electrostatic elements, new tools and methods had to be developed to study the USR beam dynamics in detail. Studies have been done into the long term beam dynamics [12], as well as into comparison between different established and purpose-written computer codes [13]. This provided a consistent explanation of some of the effects observed in electrostatic storage rings. It was found that multiple scattering of the stored ions on the atoms and molecules of the residual gas, together with intra beam scattering at higher beam intensities lead to a fast growth in beam emittance. In combination with a significant reduction of the ring acceptance because of non linear fields in electrostatic ion optical elements, experimental data was reproduced with very good accuracy. A realistic beam life time at $20 \mathrm{keV}$ and maximum beam intensity of $2 \cdot 10^{7}$ antiprotons of up to $10 \mathrm{~s}$ was determined. Other important beam parameters are summarized in the following table 1 .

Table 1: Summary of main USR parameters

\begin{tabular}{lc}
\hline \multicolumn{1}{c}{ Parameter } & \multicolumn{1}{c}{ Value } \\
\hline Energy & $300 \mathrm{keV} \rightarrow 20 \mathrm{keV}$ \\
\hline Relativistic $\beta=v / c$ & $0.025 \rightarrow 0.006$ \\
\hline Revolution frequency & $177 \mathrm{kHz} \rightarrow 46 \mathrm{kHz}$ \\
\hline Revolution time & $5.6 \mathrm{~ms} \rightarrow 32.8 \mathrm{~ms}$ \\
\hline Number of particles & $\leq 2 \cdot 10^{7}$ \\
\hline Bunch length & $1 \mathrm{~ns}-\mathrm{DC}$ beam \\
\hline Effective in-ring rates & $10^{10} \mathrm{pps}-10^{12} \mathrm{pps}$ \\
\hline $\begin{array}{l}\text { Average rate of extracted } \\
\text { antiprotons }\end{array}$ & $5 \cdot 10^{5} \mathrm{pps}-10^{6} \mathrm{pps}$ \\
\hline
\end{tabular}

\section{Slow/Fast Extraction}

The USR will provide external experiments with both, single pulses of up to $10^{7}$ antiprotons per deceleration cycle as well as quasi-continuous beams for nuclear physics-type experiments. Based on preliminary studies [14], the combined fast and slow extraction region was recently redesigned [15]. In order to accommodate both, slow and fast extraction, in the same $4 \mathrm{~m}$ long straight section of the storage ring, the parallel plate bump electrodes, the adjustable septum electrode and the electrostatic extraction channel will be used for both extraction modes. During injection and deceleration of the stored antiprotons the beam size in the straight section will be as large as $20 \mathrm{~mm}$. The growth of the radial beam size during the slow extraction process on the other hand will not exceed $7 \mathrm{~mm}$.

The extraction system consists of the following elements: two parallel plate deflectors with a gap of 60 $\mathrm{mm}$, two slightly larger parallel plate deflectors with a plate distance of $90 \mathrm{~mm}$, a $6^{\circ}$ extraction septum and a $30^{\circ}$ cylinder deflector. The electrostatic septum is located inbetween the two central bump electrodes and is displaced by $50 \mathrm{~mm}$ with respect to the central orbit. This provides sufficient free space and safety margins for the circulating, as well as the extracted beam.

During the extraction process, the beam will first be moved by a local orbit bump towards the extraction septum. In the process, the beam is first deflected by an angle between $4.5^{\circ}$ and $6^{\circ}$, before the second (larger) bump electrode returns the beam to its initial direction. The beam then runs parallel to the equilibrium orbit but is shifted away from the ring axis by $30-40 \mathrm{~mm}$ during the passage of the extraction part of the straight section. A second pair of bump electrodes then directs the beam back onto the ring axis. For fast extraction, an additional pulsed voltage of opposite polarity will be applied to the plates of the first large bump deflector so that the beam can directly enter into the septum extraction channel. Full details about the extraction process can be found in [10].

\section{Antiproton-Atom Collisions}

The study of the dynamics of collisions between antiprotons and fundamental systems such as the Helium atom, is one of the central goals of the USR. For the first time, single and double ionization interactions shall be studied by measuring kinematically complete differential cross sections to obtain the full momentum vectors of all reaction products. Using the stored antiproton beam will improve the luminosity by at least six orders of magnitude compared to single pass experiments. These experiments require the beam to be compressed to pulses of only 1-2 ns duration to allow the time structure of the beam as a trigger signal for the experiment. The scheme described below will be used to generate these short bunches.

After deceleration to $20 \mathrm{keV}$ in the USR, the beam is electron cooled in about $1 \mathrm{~s}$ to a momentum spread of $5 \cdot 10^{-4}$. This coasting beam is then adiabatically captured into stationary buckets formed by a $20 \mathrm{MHz}$ cavity, 
operating at a high harmonic mode of the revolution frequency, $h_{R F}=440$. This leads to bunches of $18 \mathrm{~ns}$ pulse width (FWHM) with a distance between individual pulses of $50 \mathrm{~ns}$. Ultra-short pulses of 1-2 ns duration are then formed by an additional $3 \beta \lambda / 2$ double drift buncher. This buncher is located at the beginning of the straight section of the USR where the reaction microscope is integrated. Bunches of $\sim 2$ ns pulse width are then formed in the symmetry point of the straight section. The USR lattice as well as all focusing electrostatic elements in the ring have been optimized to accommodate a beam with a momentum spread up to $\Delta p / p= \pm 1 \%$. In order to provide a time focus at a distance of $2 \mathrm{~m}$ downstream the phase compressor, the required amplitude of the phase compressor voltage is only $U_{\text {bunch }}=370 \mathrm{~V}$.

Once the experimental section is crossed, energy compression is mandatory. An RF phase de-compressor is therefore located next to and downstream of the reaction microscope and will mostly compensate the energy spread introduced by the buncher and limit the growth of the equilibrium momentum spread to an acceptable level. In this scenario, the energy compressor is located $2 \mathrm{~m}$ away from the reaction microscope. Full details are described in [9].

\section{BEAM DIAGNOSTICS}

The low energy, intensity and variable pulse structure of the antiproton beam in the USR from nanosecond pulses to a DC beam, require the development of new beam diagnostics methods as most conventional techniques used in medium and high energy accelerators will not work. Over the course of the past few years instrumentation for the commissioning phase of the USR with proton $/ \mathrm{H}^{-}$beams was developed, as well as specialized diagnostics for the operation with antiproton beams. A compact Faraday cup was designed, built up and tested with femto Ampere beams at energies down to 100 $\mathrm{keV}$. For non-destructive beam position determination, up to eight capacitive pick-ups (PUs) will be installed at the USR. The expected weak signals require the use of high input resistance, high gain/low noise amplifiers, together with a narrowband processing system. A prototype pickup has been developed, built up and successfully tested in a purpose-built test stand. Finally, scintillating screens and a secondary emission monitor were tested for profile measurements in close collaboration with the group of $\mathrm{P}$. Finocchiaro at INFN-LNS in Catania and preparations for measurements with low energy antiprotons at the AEgIS experiment, CERN have recently been finalized.

In addition to the above monitors, an ionization beam profile monitor relying on a supersonic gas-jet shaped into an extended thin screen is being developed. When the stored beam crosses the jet, ionization occurs. These ions are then accelerated by an electric extraction field towards an MCP amplification stage and detected via a phosphor screen and a CCD camera. Detailed investigations into the process of gas jet formation have been realized [16]. These studies helped improve the understanding of the dynamics of such jets and indicated ways to control the jet properties. The jet has been assembled at the Cockcroft Institute and first experimental tests have been done [17].

\section{ACKNOWLEDGEMENTS}

The generous support of the Helmholtz Association of National Research Centers and GSI Helmholtz Centre for Heavy Ion Research under contract VH-NG-328, the EU under contract PITN-GA-2008-215080, the Max Planck Institute for Nuclear Physics and the STFC Cockcroft Institute Core Grant No. ST/G008248/1 is acknowledged.

\section{REFERENCES}

[1] C.P. Welsch, et al., "FLAIR - A Facility for LowEnergy Antiproton and Ion Research at GSI", Hyperfine Interactions, 172 (2007) 71-80

[2] http://www.flairatfair.eu

[3] M. Niering et al., Phys. Rev. Lett. 84 (2000) 5496

[4] H. Hellwig et al., Proc. IEEE Trans. IM-19 (1970) 200

[5] L. Essen, R. W. Donaldson, M. J. Bangham and E. G. Hope, Nature 229 (1971) 110

[6] R. J. Hughes, "Antihydrogen and fundamental symmetries", Hyperfine Interactions 76 (1993) 3c

[7] C.P. Welsch, et al., "Exploring Sub-Femtosecond Correlated Dynamics with an Ultra-low Energy Electrostatic Storage Ring", AIP Conf. Proc. 796 (2005) p. 266-271

[8] C.P. Welsch, et al., "An ultra-low-energy storage ring at FLAIR", NIM A 546 (2005) 405-417

[9] A.I. Papash, C.P. Welsch, "Realization of Nanosecond Antiproton Pulses in the Ultra-low Energy Storage Ring", Nucl. Instr. and Meth. A 620 (2010)

[10] G.A. Karamishev, et al., "Study of Slow and Fast Extraction for the Ultralow Energy Storage Ring USR“, Part. Phys. Nucl. Lett. 8 (2011) 50-60

[11] A.I. Papash, et al., "An update of the USR Lattice: Towards a true multi-user experimental facility", Proc. Part. Acc. Conf., Vancouver, Canada (2009).

[12] A.I. Papash, et al., "Investigations into the beam life time in low energy storage rings", Proc. Intern. Part. Acc. Conf., New Orleans, USA (2012).

[13] D. Newton et al., "Investigations into Non-linear Beam Dynamics in Electrostatic Storage Rings", Proc. IPAC, San Sebastian, Spain (2011)

[14] P. Schmid, et al., "Preliminary Design of an Extraction Scheme for the USR", Hyperfine Interact. 194 1-3 p. 183-187 (2009)

[15] J. Harasimowicz, et al., "Scintillating Screens Sensitivity and Resolution Studies for Low Energy, Low Intensity Diagnostics", Rev. Sci. Inst. 81 (2010)

[16] M. Putignano, et al., " Numerical study on the generation of a planar supersonic gas-jet", Nucl. Instr. Meth. A 667 (2012) p. 44-52.

[17] M. Putignano, et al., Proc. BIW (2012). 